\title{
The Role of Endocytosis during Morphogenetic Signaling
}

\author{
Marcos Gonzalez-Gaitan ${ }^{1}$ and Frank Jülicher ${ }^{2}$ \\ ${ }^{1}$ Department of Biochemistry, University of Geneva, 1211 Geneva 4, Switzerland \\ ${ }^{2}$ Max-Planck Institute for the Physics of Complex Systems, 01069 Dresden, Germany \\ Correspondence: marcos.gonzalez@unige.ch; julicher@pks.mpg.de
}

Morphogens are signaling molecules that are secreted by a localized source and spread in a target tissue where they are involved in the regulation of growth and patterning. Both the activity of morphogenetic signaling and the kinetics of ligand spreading in a tissue depend on endocytosis and intracellular trafficking. Here, we review quantitative approaches to study how large-scale morphogen profiles and signals emerge in a tissue from cellular trafficking processes and endocytic pathways. Starting from the kinetics of endosomal networks, we discuss the role of cellular trafficking and receptor dynamics in the formation of morphogen gradients. These morphogen gradients scale during growth, which implies that overall tissue size influences cellular trafficking kinetics. Finally, we discuss how such morphogen profiles can be used to control tissue growth. We emphasize the role of theory in efforts to bridge between scales.

\begin{abstract}
A fundamental challenge in biology is to unAderstand how morphologies and complex patterns form in multicellular systems by the collective organization of many cells. Cells divide and undergo apoptosis, and they communicate via signaling pathways that use molecules as information carriers. In tissues, large-scale patterns of gene expression emerge from the coordinated signaling activity and response of many cells. The establishment of such patterns is often guided by long-range concentration profiles of morphogens. Cell divisions and cell rearrangements must be coordinated over large distances to achieve specific tissue sizes and shapes. To unravel how molecular processes and interactions can eventually be responsible
\end{abstract}

for the formation of structures and patterns in tissues during development, it is important to study processes at different scales and understand how different levels of organization are connected. Such an approach becomes strongest if it involves a combination of quantitative experimental studies with theory.

In the present article, we discuss several such approaches on different scales with a particular emphasis on theory. Starting from the kinetic and dynamic properties of endosomal networks inside a cell, we discuss transport processes in a tissue that can be related to kinetic trafficking parameters. Such transport processes are then responsible for the formation of graded morphogen concentration profiles. To permit

Editors: Sandra L. Schmid, Alexander Sorkin, and Marino Zerial

Additional Perspectives on Endocytosis available at www.cshperspectives.org

Copyright (C) 2014 Cold Spring Harbor Laboratory Press; all rights reserved; doi: 10.1101/cshperspect.a016881

Cite this article as Cold Spring Harb Perspect Biol 2014;6:a016881 
scalable patterns in tissues of different sizes, it has been suggested that morphogen gradients scale during growth. This can be achieved on the tissue level by feedback systems that are sensitive to tissue size and regulate, for example, morphogen degradation. Finally, morphogen gradients that scale with tissue size can provide a system to robustly organize cell division in a large tissue and generate homogeneous growth. Theory can play an important role to bridge scales and understand how molecular and cellular processes can control pattern formation and tissue growth on larger scales.

Morphogens are signaling molecules that are secreted in specific regions of developing tissues and can induce signaling activity far from their source. They typically form graded concentration profiles and therefore endow cells with positional information (cells can obtain information about their position in a tissue). Thus, they can guide cells to differentiate into complex morphological patterns. Morphogens also control cell growth and cell division. Because they control both patterning and growth, they may play a key role to coordinate these two processes. Such coordination is important because the size of morphological patterns must adjust during growth, whereas growth influences such patterns. A well-studied morphogen is Decapentaplegic (Dpp), which controls morphogenesis in the imaginal wing disc of developing Drosophila. Consequently, mutations in Dpp or defects in the trafficking pathways that control its graded concentration profiles and signaling affect the formation and structure of the adult wing.

The study of morphogens was traditionally approached from a genetic perspective: Which gene products behave like morphogens? Which mutants affect patterning and growth? The realization that morphogens typically operate by a gradient of concentration raised the question of how morphogen gradients are generated. It became clear that the cellular trafficking of morphogens is a key issue for the generation of morphogen profiles. Morphogens are secreted ligands that bind receptors in the plasma membrane. The secretion of the ligands and the concentrations of receptor, ligand, and receptor/ ligand complex at the plasma membrane are governed by their trafficking in the cell by vesicular transport. In particular, it was shown that trafficking through the endocytic pathway has an important impact on the formation of morphogen gradients (reviewed in Gonzalez-Gaitan 2003; see Bökel and Brand 2014). This is, to a large extent, how the cells respond to morphogens and contribute to set their local concentrations. To understand functions of morphogens in a tissue, we need to study how the gradient is formed. This, in turn, requires insights into morphogen trafficking through the endocytic pathway. The problem of morphogen behavior, therefore, becomes a problem spanning several levels of complexity: the organ level, the tissue level, the cell level, the organelle level, and the molecular level. Theoretical approaches motivated by physics combined with quantitative experimental approaches provide an ideal framework to understand how these different levels of complexity are intertwined.

Two recent discoveries highlighted such integration. (1) The observation that profiles of the morphogen Dpp scale during growth, which implies that the rate of Dpp degradation mediated by the endocytic pathway of each of the cells in the tissue depends on the size of the overall tissue. This suggests that two levels of complexity are linked because cellular trafficking receives cues about the global tissue size. (2) As a result of the changes of the degradation rate that leads to gradient scaling, cells receive an increasing level of signaling. This, in turn, can be used by the cells to decide when to divide. This regulation again involves two levels of complexity because regulation at the endocytic pathway determines the growth properties of the tissue and, ultimately, its final size.

In the following, we discuss quantitative approaches to study cellular signaling processes on different scales. Here, the aim is to understand how patterns on large scales can emerge during development from molecular processes and signaling pathways that involve endocytosis and cellular trafficking. We begin by describing trafficking of ligands in the endocytic pathway. We then consider the situation of a morphogen ligand and its impact in gradient formation. Subsequently, we discuss how gradient scaling 
might be realized. Finally, we discuss how such scaling processes play an important role in the regulation of morphogenetic growth.

\section{CELLULAR TRAFFICKING}

Endocytosis leads to the import of cargo molecules, which are then distributed into a series of endosomes with characteristic morphological and biochemical properties (Fig. 1A) (Mellman 1996; van der Goot and Gruenberg 2006; see also Klumperman and Raposo 2014; Mayor et al. 2014). These endosomes form a dynamical network in the cell in which cargo is exchanged and redistributed by fusion and fission events. After endocytosis, cargo enters early endosomes with distinct membrane properties organized by the small GTPase Rab5 (Zerial and McBride 2001; Rink et al. 2005; Wandinger-Ness and Zerial 2014). Cargo can then be recycled to the plasma membrane via recycling endosomes or degraded by conversion of early endosomes to late endosomes characterized by Rab7 (Dunn et al. 1989; Rink et al. 2005).

Traditionally, the kinetics of signaling systems in a cell is characterized by the temporal changes of the average cellular levels of activated signaling molecules regardless of their location. However, such signals are processed and stored in intracellular vesicular networks that consist of different types of endosomes and affect the signals in distinct manners (Di Fiore and von Zastrow 2014). To understand the dynamics of cellular signaling processes, the discrete nature of the endosomal network needs to be taken into account.

Recently, we have developed a quantitative approach to understand how the kinetics of the overall endosomal network emerges from the interactions of individual endosomes (Foret et al. 2012). Fluorescently labeled Rab5 expressed at endogenous levels was used as a marker to monitor early endosomes. Cells were exposed to a solution containing fluorescently

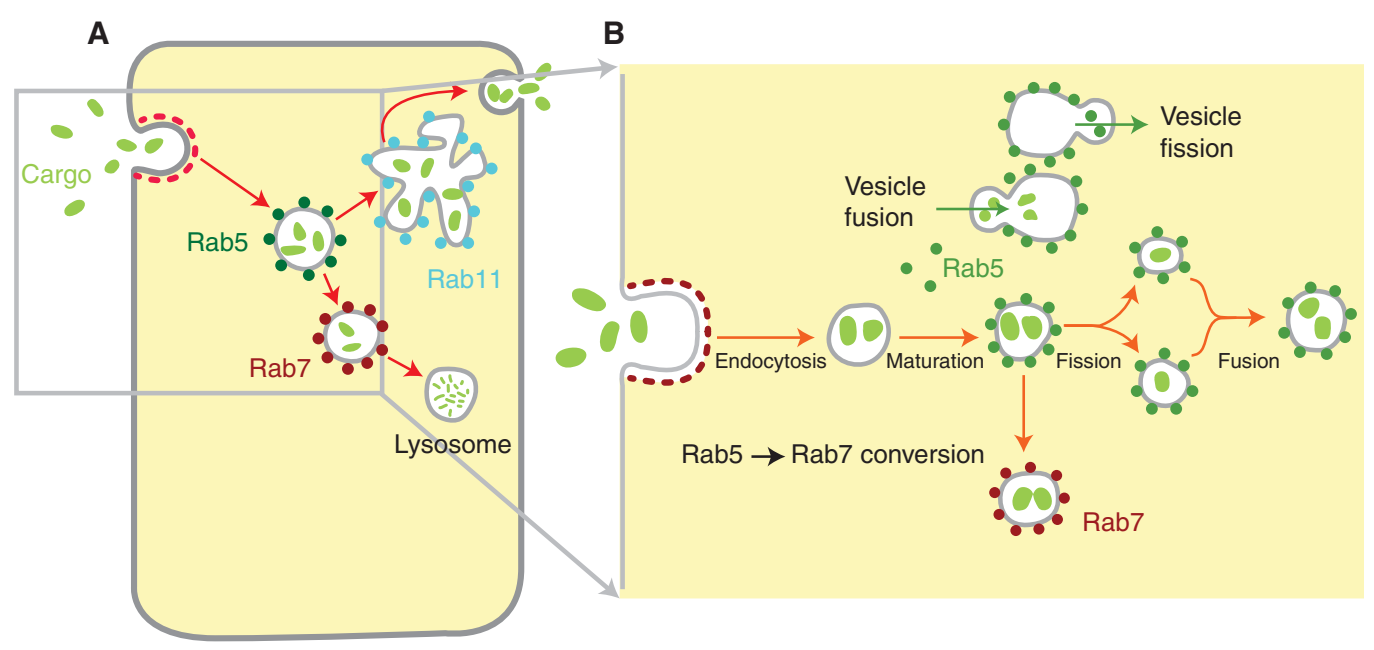

Figure 1. Cargo trafficking through the endocytic pathway. $(A)$ The endocytic pathway. Extracellular cargo (light green blobs) is internalized by endocytosis mediated by clathrin (red bars). Cargo then enters the early endosomes characterized by the small GTPase Rab5. Rab5 early endosomes convert into late endosomes by exchanging Rab5 by Rab7. Rab7 late endosomes fuse with lysosomes to degrade cargo. Cargo from the Rab5 early endosomes can also traffic subsequently into the recycling endosome characterized by Rab11 from where cargo can be recycled by being targeted back to the plasma membrane. (B) Dynamic events shaping the early endosome network. After internalization, endocytic vesicles can fuse with early endosomes, thereby bringing cargo into the endosomal network. Rab5 endosomes can receive more cargo by homotypic fusion with other endosomes. They can lose cargo by homotypic fission. Cargo can leave the early endosome network by heterotypic fission of or conversion of early to late endosomes. 
labeled low-density lipoprotein, a cargo that, upon internalization, enters the endosomal network. We then used high resolution automated imaging analysis to track and generate large data sets of the dynamic behavior of rab5- and cargo-containing endosomes. This permitted us to measure the distribution $n(s)$ of cargo amounts in early endosomes. Here, $n(s) \Delta s$ is the number of endosomes carrying a cargo amount between $s$ and $s+\Delta s$ determined by the fluorescence intensity of labeled cargo (Fig. $1 \mathrm{~A}, \mathrm{~B})$. This quantitative study of endosome network dynamics was based on a theoretical approach that allowed us to derive a dynamic equation for cargo distributions in endosomes. It could be shown that key features of the shape and the time dependence of this cargo distribution function could be explained by the four basic processes by which endosomes exchange cargo, namely, cargo influx into the endosomal network, homotypic endosome fusion, endosome fission, and early endosome conversion to late endosomes (Fig. 1B).

This theory describes how cargo gets distributed over time in a large number of endosomes. If the cargo source was present over long times, the distribution reaches a steady state in which the cargo distribution has a characteristic shape. This steady-state distribution shows power-law behaviors. This implies that the cargo amounts in single endosomes can vary over wide ranges within the same endosomal network. This is a consequence of redistribution of cargo by homotypic endosome fusion. Quantification of the cargo distribution provides information about kinetic parameters of endosomes such as cargo influx, endosome fusion rate, and endosome lifetime. The steady state reveals only ratios of these parameters. Quantification of the full-time course of the cargo distribution in cells shows that this time dependence is captured quantitatively by the theory. From this analysis, we estimated at steady state that the average time between two fusion events was $\sim 3 \mathrm{~min}$. Endosome lifetime was $\sim 11 \mathrm{~min}$.

Furthermore, this approach could be used to study how perturbations of the system influence endosome kinetic parameters and give rise to altered cargo dynamics and distributions in the endosomal network. In perturbation experiments, the Rab5 effector and endosomal-tethering factor EEA-1 was depleted using RNA interference. EEA-1 localizes to the membrane of early endosomes and acts as a tethering factor, a precondition for endosome fusion (Christoforidis et al. 1999; Gautreau et al. 2014). Quantification of cargo distributions in cells with fluorescently labeled Rab5 revealed significant changes of cargo distributions as compared with control experiments, which can be explained by altered values of kinetic parameters. Analyzing the data using the theory to estimate endosome kinetic parameters revealed that knockdown of EEA1 led to a reduction of cargo influx and rate of endosome fusion. This result is consistent with the known role for EEA1 in fusion of endocytic vesicles with early endosomes (Horiuchi et al. 1997) and homotypic fusion of endosomes (Simonsen et al. 1998). Therefore, it shows that out of the many trafficking steps that could be altered in principle (Fig. 1A,B), the theory allows determining those that are altered on the functional perturbations (either genetics or pharmacological) by analyzing the dynamic cargo distributions in endosomes.

Together, these data suggest that endosome dynamics (fission, fusion, and maturation) determine their ability to sort and concentrate cargo molecules; when the cargo is a morphogen, these processes help to establish its concentration and signaling activities within individual cells.

\section{MORPHOGEN GRADIENTS ON THE TISSUE LEVEL}

In developing tissues, cells in a discrete region of the primordium secrete morphogens, which can stimulate a signal transduction cascade in distant cells by binding to specific receptors on the cell surface (Fig. 2A,B) (Turing 1952; Wolpert 1969). Morphogen molecules are released from a localized source (Fig. 2B) and diffuse in the extracellular space. Morphogens are recognized by receptors on the target cells and the receptor-ligand complexes undergo endocytosis (Fig. 2C, $k_{\text {int }}$ ), and are then sorted 

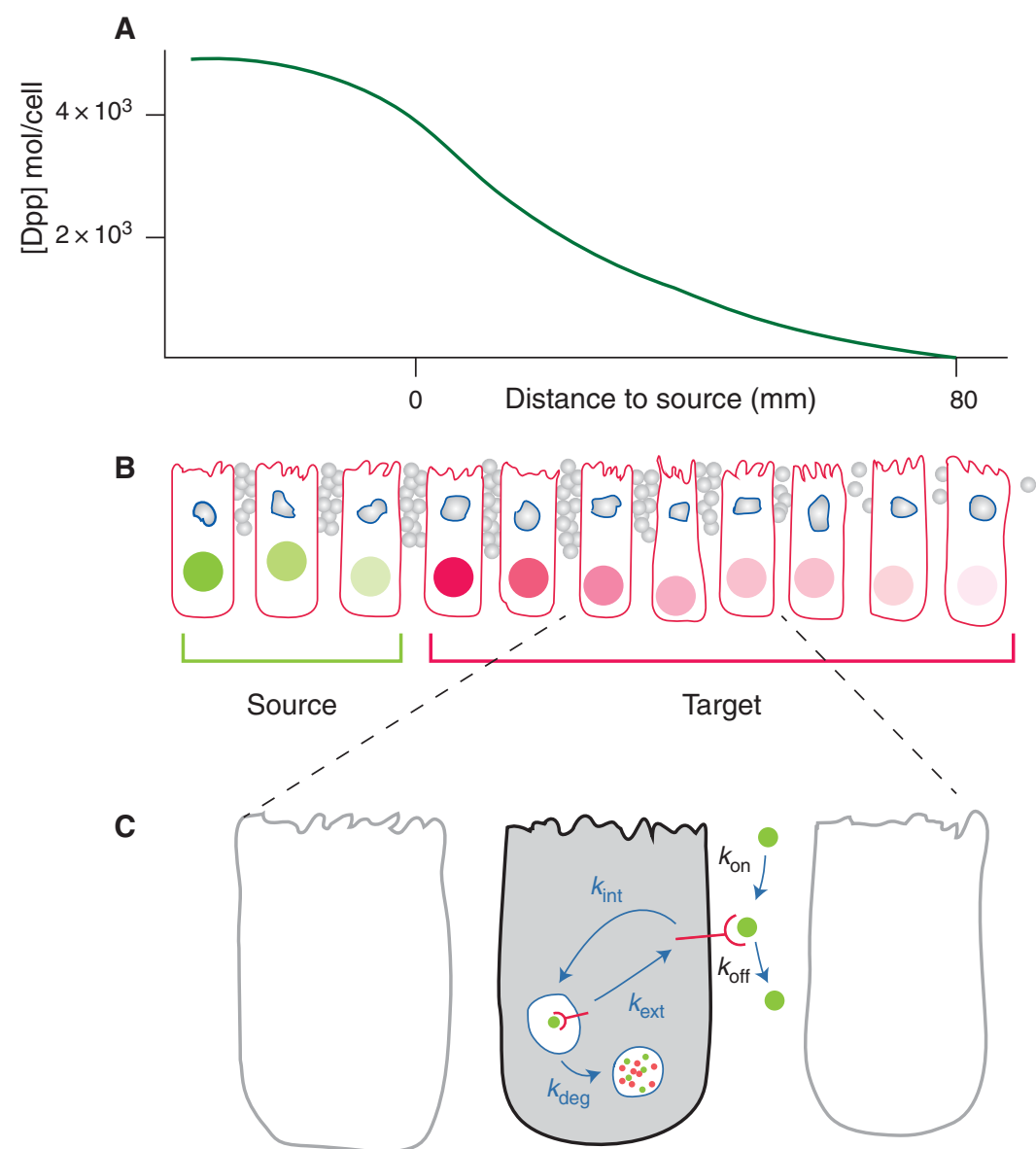

Figure 2. Morphogen gradients and the endocytic pathway. (A) The morphogen Dpp in the developing wing forms a graded concentration profile that decreases with increasing distance from the source. $(B)$ Source cells secrete the morphogen that diffuses in the target tissue. $(C)$ Diffusing morphogens bind to receptors in target cells with a characteristic binding rate $\left(k_{\text {on }}\right)$ from where they are released with a characteristic rate $\left(k_{\text {off }}\right)$. On ligand binding, the receptor is internalized with a characteristic internalization rate $\left(k_{\text {int }}\right)$ into endosomes. From there the ligand can be degraded $\left(k_{\mathrm{deg}}\right)$ or recycled, and externalized $\left(k_{\mathrm{ext}}\right)$.

and transported in the cell by endosomal networks (Gonzalez-Gaitan 2003). After entering the cell, receptor-ligand complexes are targeted to early endosomes. When such endosomes convert to late endosomes, the ligands will be targeted for degradation (Fig. 2C, $k_{\mathrm{deg}}$ ). Alternatively, receptor-ligand complexes can be recycled to the cell surface from where the ligands may be liberated and move to a different cell (Fig. 2C, $k_{\text {ext }}$ ) (Entchev et al. 2000). Release of the ligand from the receptor could also happen in the recycling endosomes in a $\mathrm{pH}$-dependent process.
Thus, morphogen transport is influenced by receptor binding, the dynamics of receptor-ligand complexes on the cell surface, and receptor and ligand trafficking in endosomal networks (Fig. 2C) (Affolter and Basler 2007). Recycling of morphogens to the cell surface can play an important role for their transport as it provides a means for long-range transport from cell to cell in the tissue via endocytosis and recycling, a process called transcytosis (Entchev et al. 2000). The balance between diffusion, degradation, and recycling can generate a graded concentration profile in the target field, termed 
"gradient" by developmental biologists. This graded concentration elicits a graded level of activation of the downstream pathway, which results in different transcriptional responses depending on the concentration of the morphogen. The morphogen profile, therefore, can endow cells with positional information.

An important question is to understand how the transport behavior of ligands on the tissue scale depends on the kinetics of cellular trafficking processes. This has been studied by using endocytic mutants such as conditional alleles (thermosensitive mutants) or mosaic situations in combination with quantitative studies (Kruse et al. 2004). Theoretical approaches can be then be applied to study how transport properties in a collection of cells emerge from processes at the cell scale.

Starting with the kinetics of elementary trafficking events, we can systematically derive dynamic equations describing morphogen transport in a tissue over distances of many cells (Bollenbach et al. 2007). Such transport equations on the tissue scale involve the unbiased spreading of molecules in the tissue, which can be characterized by an effective diffusion coefficient $D$. Effective diffusion can occur in the case of transcytosis even if, microscopically, ligand molecules do not efficiently diffuse in the space between cells. This implies that internalization of receptor-ligand complexes and subsequent recycling to the cell surface together with ligands transiting across the narrow gap between adhering cells provides a means of diffusive transport. The effective diffusion coefficient $D$ can therefore, in general, be quite different from molecular diffusion coefficients and significantly affected by receptor binding and receptor trafficking in the cell. In particular, transcytosis allows for long-range transport in a tissue if extracellular diffusion is inefficient.

A second important rate is the effective degradation rate $k$. Degradation can happen in the extracellular space or by the degradation of molecules in the cell. Typically, degradation occurs after the early endosomes carrying the ligands convert into late endosomes. Finally, an important kinetic parameter is the source strength $v$, which is defined as the number of molecules secreted by a source cell per cellular area and unit time. The effective diffusion coefficient and the effective degradation rate can be expressed in terms of cellular trafficking rates, receptor binding and internalization rates, and rates of extracellular diffusion (Bollenbach et al. 2007).

Based on these concepts, the ligand profiles in a tissue can then be captured by a sourcediffusion-degradation equation (Bollenbach et al. 2005). In the case of position-independent diffusion coefficient and degradation rate, steady-state profiles show an exponential decay as $C(x)=C_{0} e^{-x / \lambda}$, in which $C(x)$ is the morphogen (area) concentration at a distance $x$ from the source (Fig. 2D). The decay length $(\lambda)$ of the profile depends on the diffusion coefficient $(D)$ and the degradation rate $(k)$ as $\lambda=\sqrt{(D / k)}$. Furthermore, the amplitude of the profile $\left(C_{0}\right)$ depends on the morphogen pro duction rate $(v)$ and the width of the source of production $(w)$ according to $C_{0}=$ $(v / 2 k)\left(1-e^{-w / \lambda}\right)$. Note, again, that the effective diffusion coefficient $D$ and the effective degradation rate $k$ are tissue level parameters that themselves depend on cellular parameters, such as the kinetics of cellular trafficking of receptors and ligands as mentioned above.

The transport coefficients of ligands in a tissue can be determined using fluorescence recovery after photobleaching (FRAP) assays. In such experiments, green fluorescent protein (GFP)-labeled ligands are bleached and the recovery of fluorescence is detected. By fitting theoretical recovery curves to experimental data, the effective diffusion coefficient, effective degradation rate, mobile fraction, as well as the source strength have been determined. This approach led to the estimates for the diffusion coefficient of $D=0.1 \mathrm{\mu m}^{2} / \mathrm{sec}$, and a degradation rate $k=2.5 \times 10^{-4} \mathrm{sec}^{-1}$, corresponding to a half-life of Dpp molecules of $45 \mathrm{~min}$. The production rate in the source cells was estimated as $v=3$ molecules per cell per second. Using temperature-sensitive mutants of dynamin, which suppress endocytosis, it was shown that the effective diffusion coefficient of Dpp in the wing imaginal disk of Drosophila significantly depends on endocytosis (Kicheva et al. 2007). 
This suggested that Dpp is transported in a process involving transcytosis. In contrast, in the case of wingless $(\mathrm{Wg})$, another ligand that is distributed as a graded profile (it is, however, not a bona fide morphogen), such an effect could not be seen, indicating that Wg is transported extracellularly (Kicheva et al. 2007).

More recently, fluorescence correlation spectroscopy (FCS) has been used to estimate the diffusion coefficient of morphogens (Yu et al. 2009). It should be noted, however, that FCS probes molecular movements at short timescales and within the illumination volume with a diameter of about 200-500 nm (Kicheva et al. 2012). Therefore, FCS does not provide estimates of effective diffusion coefficients of ligands in a tissue over larger scales, but characterizes diffusion on subcellular scales (see Kicheva et al. 2012). Also, it may be difficult to estimate the diffusion coefficient in the extracellular space using FCS. The extracellular space can be as small as 10-20 nm, whereas the illumination volume for FCS extends into the adjacent cells (Kicheva et al. 2012). Therefore, characterization of morphogen diffusion by FCS at the local scale of 200-500 nm can indeed provide interesting information. However, it does not capture, by itself, the spreading kinetics at the tissue scale. Indeed the values of the diffusion coefficient obtained by FCS, if applied at the tissue scale, generate a conundrum when comparing with the measured receptor binding rates and kinetic rates of endocytosis.

Indeed, based on FCS experiments, it has been suggested that $60 \%$ of the extracellular Dpp molecules move with a diffusion coefficient $D=20 \mu \mathrm{m}^{2} / \mathrm{sec}$ (Zhou et al. 2012). If such fast diffusion were relevant on the tissue scale, it would make implausible a scenario of transport of morphogen by intracellular trafficking and recycling (in the scale of many minutes), and instead would suggest transport by rapid extracellular diffusion. Because the observed gradient-decay length is $\sim 20 \mu \mathrm{m}$, this value of the diffusion coefficient would imply a fast receptor-binding kinetics (capture) with a characteristic time of $\sim 15 \mathrm{sec}$ at the end of development when these measurements were made. Because gradients scale with the size of the tissue (which is mediated by the turnover rate of Dpp; see below Wartlick et al. 2011b), early in development these binding rates should be two orders of magnitude larger (as compared with late stages), corresponding to binding times of $\sim 0.15 \mathrm{sec}$. Such rapid binding is, however, unrealistic for receptor-ligand complexes. It would also imply that only a very small fraction of Dpp molecules could diffuse freely. Indeed, comparing this time scale of binding with the time scale of internalization $(\sim 189 \mathrm{sec})$ (Taylor et al. 2011), this implies that only at most $8 \%$ (i.e., $15 / 189$; or, in early discs, $0.08 \%$, corresponding to $0.15 / 189$ ) of the Dpp molecules outside the cell would be freely diffusing instead of $60 \%$ as reported (Zhou et al. 2012).

This conundrum could arise from the fact that the diffusion coefficient determined by FCS is a microscopic diffusion coefficient and not the effective diffusion coefficient that characterizes transport at the tissue scale; although the microscopic diffusion of Dpp could be fast, rapid capture of Dpp by receptors would hamper its spreading in the tissue. Long-range transport of the morphogen in the tissue could be facilitated by internalization, intracellular trafficking, and recycling of the ligand.

It has also been suggested that Dpp spreads by restricted extracellular diffusion (Belenkaya et al. 2004). Restricted diffusion means that the molecule spreads in a narrow extracellular space where it may bind to extracellular matrix proteins such as heparan sulfate proteoglycans and other glypican family members. As a consequence, the effective diffusion coefficient may be much smaller than the one estimated from free diffusion in a solvent. Two effects may be important here. (1) The extracellular space between cells may provide narrow channels forcing molecules on complex and extended trajectories (Rusakov and Kullmann 1998), thereby reducing the effective diffusion coefficient. (2) Binding to glypicans on the cell surface could hamper diffusion. Restricted diffusion might account for the small values of diffusion coefficients observed by FRAP experiments, but would not be expected to lead to a dependence of the diffusion coefficient on dynamin activity as observed (Kicheva et al. 2007). 
M. Gonzalez-Gaitan and F. Jülicher

\section{SCALING OF MORPHOGEN GRADIENTS BY ENDOCYTIC PATHWAYS}

Regardless of the actual mechanism of morphogen spreading and gradient formation, the spatial profile of the morphogen in the developing tissue depends on three key parameters described above: (1) its rate of production at the source, which depends on transcription, translation, and secretion; (2) its mobility in the tissue characterized by the effective diffusion coefficient; and (3) its rate of turnover (degradation). Ultimately, these three properties characterized by the parameters $D, k, v$ are dominated mainly by the trafficking behavior of the morphogen in the secretory and endocytic pathways. Before their differentiation, cells in a growing, developing epithelium have similar properties at different positions and belong to a particular, single-cell type. Therefore, in principle, their trafficking properties would also be approximately homogeneous in space and, a priori, no changes in the properties of the morphogen gradient are expected as the tissue grows. If cellular properties do not change during tissue growth, the decay length, which depends on the effective diffusion coefficient and the degradation rate, would be constant in time and the gradient amplitude would undergo only minor changes due, for example, to a change in the width $(w)$ of the source of morphogen production.

It has, however, been long known that morphological patterns, which are processes that are guided by morphogen gradients, can scale proportionally to the size of tissues. In a classical experiment, Spemann and Mangold (1924) showed that on cutting a frog embryo in half, one of the halves developed into an animal with half the size, but normal morphological proportions. Ever since, morphogen gradient scaling has been shown directly or indirectly in a number of instances (Teleman and Cohen 2000; BenZvi et al. 2008; Wartlick et al. 2011b). In particular, in the fly, it was shown that the decay lengths of the Dpp gradients in the imaginal discs that will give rise to the wing and the haltere (another appendage in the fly) are proportional to the growing length of these tissues during their growth phase (Fig. 3A) (Wartlick et al. 2011b). This implies that as the tissue grows the profile of the morphogen gradients would collapse into a single curve if the gradients are normalized to tissue size $(x / L)$ and maximal concentration $\left(C_{\text {cell }} / C_{0}\right)$ (Fig. 3B). The expansion of the decay length of the gradient is not due to the fact that Dpp molecules are long lived and carried away from the source as the cells divide, a phenomenon termed "advection." Advection could, in principle, "stretch" the gradient during growth, whereas the ligand concentration would drop as levels get diluted by growth. Because the half-life of Dpp is more than an order of magnitude shorter than the duration of the cell cycle (Wartlick et al. 2011b), stretching by advection is negligible for the Dpp gradient. Also, the fact that the gradient amplitude increases during growth (Fig. 3A,C) suggests that dilution effects are not important. These observations imply that the gradient shape adjusts quickly to the growing tissue, and Dpp molecules are degraded before they can be transported away by tissue growth (i.e., advection of morphogen molecules by tissue growth is negligible).

What then causes the increase in the decay length and thereby the expansion of the gradient? In steady state, the decay length of the Dpp gradient depends on the diffusion coefficient and the degradation rate as $\lambda=\sqrt{D / k}$. The expansion of the decay length should therefore be due to either an increase in its diffusion coefficient or a decrease of the degradation rate. In spite of the fact that imaginal disc cells are undifferentiated and look unchanged during the growth of the tissue, either their ability to degrade Dpp or efficiency to spread it from cell to cell must change to account for a change in the decay length. By means of FRAP assays, in which the kinetics of recovery of the GFP-Dpp fluorescence in bleached territories is measured to determine $D, k$, and $v$, it was found that the changes in the diffusion coefficient and the production rate were not very large. In contrast, the changes in the degradation rate were very large during the growth phase and they could indeed account for the scaling of the decay length of the Dpp gradient (Fig. 3D) (Wartlick et al. 2011b). 
A
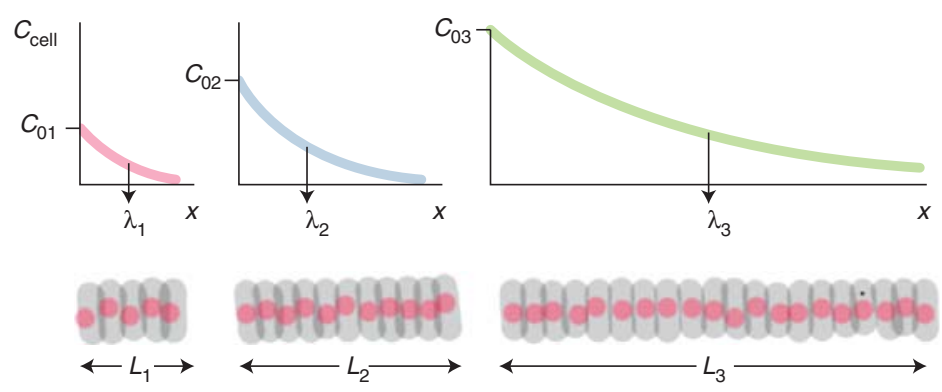

B

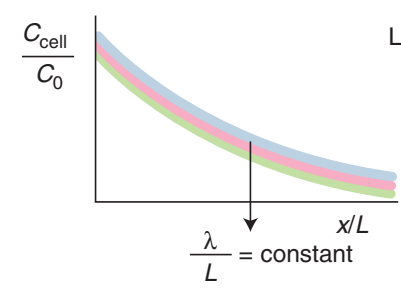

C

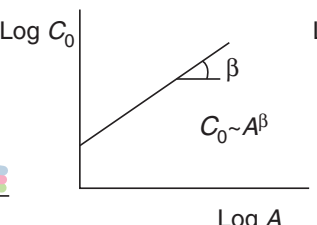

$\log A$
D

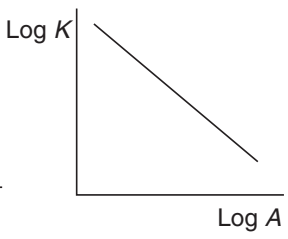

E
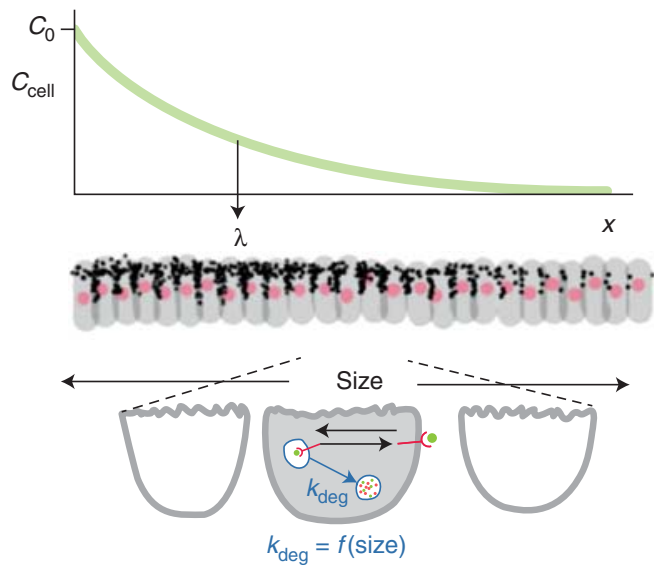

Figure 3. Gradient scaling and growth control. (A) As the tissue grows and its size $L$ becomes bigger $\left(L_{1}, L_{2}, L_{3}\right)$, both the gradient-decay length $\left(\lambda_{1}, \lambda_{2}, \lambda_{3}\right)$ and amplitude $\left(C_{01}, C_{02}, C_{03}\right)$ increase. $(B)$ Gradient scaling. The shape of the gradient profile is the same (collapse) if the gradients are normalized to relative position $(x / L)$ and relative concentration $\left(C_{\text {cell }} / C_{0}\right)$. Therefore, $\lambda / L$ is constant. $(C)$ The amplitude of the gradient is related to the size of the tissue by a power law. Note that here the area $(A)$ of the tissue is proportional to the square of its length $\left(L_{2}\right.$, i.e., growth is approximately isotropic). (D) Scaling of the gradient is caused by a decrease in the degradation rate $(k)$ of Dpp that is related to the area of the tissue by a power law. (E) Scaling is possible because each cell in the tissue sets the degradation rate of Dpp (which is mediated by the properties of Dpp endocytic trafficking) as a function of the size of the tissue.

What controls, then, the degradation rate of Dpp? Dpp is internalized into cells by endocytosis dependent on the GTPase dynamin, which controls the fission of endocytic pits at the plasma membrane (Entchev et al. 2000; Kruse et al. 2004; Kicheva et al. 2007; see also Johannes et al.
2014; Merrifield and Kaksonen 2014). Downstream, Dpp traffics through the endosomal system, including the Rab5 early endosome and the Rab1 1 recycling endosome (see Wandinger-Ness and Zerial 2014). Dpp also traffics into the Rab7 late endosomes where it is degraded (Entchev 
et al. 2000; E Entchev, A Schwabedissen, and M Gonzalez-Gaitan, unpubl.). This implies that Dpp degradation involves the endocytic pathway. Furthermore, mosaic analysis and FRAP experiments using thermosensitive dynamin mutants showed that traffic through the endocytic pathway is essential for Dpp spreading; the rates of endocytosis determine both the effective diffusion coefficient and degradation rates of Dpp (Kicheva et al. 2007). The fact that Dpp degradation is mediated by its endocytosis implies that scaling of the Dpp gradient is mediated by the size-dependent regulation of the lysosomal degradation of Dpp (Fig. 3E). This poses the question: "How could the endosomal system in single cells within the growing organ detect the size of the whole tissue to adjust the degradation rate?"

Recently, a theoretical mechanism to mediate the scaling of morphogen gradients with the growing size of developing tissues was proposed (Ben-Zvi and Barkai 2010; Ben-Zvi et al. 2011): the expansion-repression mechanism. The model postulates the existence of a molecule, called expander, with four fundamental properties: (1) the expander reduces the degradation rate of the morphogen, (2) it is a secreted factor, (3) it has a high diffusion coefficient and low degradation rate (it does not make a spatial gradient when secreted from a local source), and (4) its transcription in the tissue is repressed by the morphogen, therefore, it is only expressed at the edge of the disk in which the morphogen level is low.

These four properties of the expander could allow it to mediate morphogen gradient expansion in proportion with the size of the tissue (for a further discussion of this, see Wartlick et al. 2011a). Indeed, in a small piece of tissue, a wide morphogen gradient would repress expression of the expander. Tissue growth would generate cells at the edge of the organ to be far from the source of the gradient and therefore, because of a low morphogen concentration, to escape morphogen repression of the expander. Expression of the expander from the edge and rapid spreading in the tissue would reduce the degradation of the morphogen, thereby causing an increase in the decay length and expansion of the morphogen gradient until high levels of the morphogen at the edge would again repress expression of the expander. By means of a feedback loop, the gradient remains as large as the tissue in which it is deployed.

A secreted molecule, Pentagone, has been implicated in Dpp gradient scaling (Vuilleumier et al. 2010; Ben-Zvi et al. 2011; Hamaratoglu et al. 2011). In Pentagone mutants, the decay length of the signaling gradient of Dpp fails to scale; it remains constant during the growth phase of the wing imaginal disc (Vuilleumier et al. 2010; Ben-Zvi et al. 2011; Hamaratoglu et al. 2011). Interestingly, Pentagone was shown to be repressed by Dpp signaling, secreted, and spread from its source of production (Vuilleumier et al. 2010). Furthermore, Pentagone antagonizes Dpp degradation; the turnover rate of Dpp is decreased in Pentagone mutants. In addition, Pentagone binds Dally, a heparin sulfate proteoglycan (HSPG) that itself binds Dpp and has been proposed to control Dpp turnover. This feature is reminiscent of the characteristics of the expander as proposed in the expansion-repression mechanism, but the exact role of Pentagone in gradient scaling is still unclear. Also not known is the molecular/cell biological basis by which Pentagone, possibly through HSPG molecules, could control the trafficking of Dpp to adjust its degradation rate.

Another mechanism for scaling that has been proposed is expander dilution (Wartlick et al. 2011a,b). Scaling of the gradient by reducing the degradation rate implies that the half-life of Dpp doubles during each cell generation as the tissue grows; the half-life of Dpp in mother cells is half that of the daughter cells after mitosis. This is consistent with a scenario in which an expander that now promotes the degradation of Dpp is a long-lived molecule that diffuses rapidly in the tissue and is diluted by cell growth. However, Pentagone does have an antagonistic effect on the degradation rate and, therefore, has properties that differ from those of the expander in an expander dilution-scaling mechanism.

In summary, gradient scaling requires a fine-tuning of the lysosomal degradation of Dpp in proliferating cells, in which the endocytic trafficking properties of the ligand are changed to adjust to the size of the growing 
tissue. A key secreted molecule, Pentagone is implicated in the regulation of lysosomal degradation of Dpp to achieve scaling. Although the expansion-repression mechanism is an attractive proposal to explain scaling, it is still unclear whether Pentagone operates according to this scenario or whether other scenarios have to be considered. It is also not clear what the molecular cell biology of Pentagone-mediated control of Dpp degradation is.

In any case, scaling of the decay length can play an important role in achieving the proportioned patterning of morphological features according to the final size of the tissue. A simple rescaling of morphogen gradients occurs in situations in which the amplitude $C_{0}$ is unaffected when the decay length $\lambda$ is scaled with the length of the tissue $(\lambda / L=$ constant, in which $L$ is the width of the tissue). However, a decrease of Dpp degradation rate would cause, in principle, an increase on the amplitude because $C_{0}$ also depends on degradation by $C_{0}=$ $(v / 2 k)\left(1-e^{-w / \lambda}\right)$.

\section{MORPHOGENETIC GROWTH CONTROL AT THE ENDOCYTIC PATHWAY}

By considering the changes of the amplitude of a morphogen gradient as the tissue increases in size, a novel mechanism of growth control by morphogens was uncovered. Morphogen gradients endow cells with positional information necessary for patterning morphological structures in developing tissues (reviewed in Affolter and Basler 2007). But morphogens are also known to control growth; in Dpp mutants, the imaginal discs do not grow (hence its name, Decapentaplegic, the 15-disc-derived appendages do not grow) and ectopic expression of Dpp in a patch of cells within the developing wing leads to the growth of a winglet around the expressing cells (Zecca et al. 1996). The conundrum in the field was that Dpp, which controls growth, is distributed as a spatial gradient in the tissue, but the growth rates are homogenous; all cells divide with the same average cell-cycle duration, regardless of the concentration of Dpp that they perceive in the gradient. How can a gradient of Dpp regulate homogeneous growth?
Several models were proposed to resolve this conundrum. One suggestion is that it is not the absolute amount of the morphogen, but its gradient (reflecting the differences between adjacent cells) that controls growth. In other proposed scenarios, heterogeneous mechanical stresses in the periphery of the gradient could promote growth and a compensating Dpp signal, which is maximal at the source of production in the center, would balance inhomogeneous effects of stress to generate homogenous growth (reviewed in Wartlick et al. 2011a). In a model proposed recently, it is the change in Dpp signaling over time that controls growth (Wartlick et al. 2011a,b).

Quantification of Dpp gradients in the wing imaginal disc reveals not only that that the Dpp profile scales but also that the gradient amplitude increases with the size of the growing tissue according to a power law: $C_{0}(t) \sim A(t)^{\alpha / \ln 2}$, in which $C_{0}(t)$ is the gradient amplitude and $A(t)$ the area of the growing tissue at a particular time $t$ during development (Fig. 3C; with $\beta=$ $\alpha / \ln 2)$. The exponent $\beta=\alpha / \ln 2$ is constant with $\alpha=0.4$. (Wartlick et al. 2011b). This observation led to the proposal that the increase in the amplitude of the gradient, which results from the lysosomal degradation-dependent scaling of the gradient, underlies the mechanism by which tissue controls its growth until it attains its final size (Wartlick et al. 2011a,b).

The control of lysosomal degradation of Dpp described above mediates gradient scaling, and scaling of the gradient in the growing tissue implies that if the cellular concentration of Dpp is normalized to the gradient amplitude $\left(C_{\text {cell }} /\right.$ $\left.C_{0}\right)$ and the cell position considered relative to the length of the tissue $\left(x_{\text {cell }} / L\right)$, then all spatial gradient profiles collapse in a single curve (Fig. $3 \mathrm{~A}, \mathrm{~B})$. Furthermore, homogeneous growth in the tissue implies that the relative position of cells remains constant as the tissue grows, i.e., $x_{\text {cell }} / L=$ constant. Therefore, the concepts of both scaling and gradient collapse mean that for each cell in the tissue, $C_{\text {cell }} / C_{0}$ is constant over time. Thus, the increase in concentration of Dpp in each cell is proportional to the increase of the amplitude of the gradient (Fig. 3C), which increases because of gradient scaling. 
Gradient scaling implies that $C_{0}$ increases. Indeed, such an increase was experimentally observed and could be described as a power law $\left(C_{0}(t) \sim A(t)^{\alpha / \ln 2}\right)$ as discussed above (Fig. 3C) (Wartlick et al. 2011b). This power law was found when considering the gradient of the ligand, but was also observed when a signaling readout of the morphogen was considered. For the signaling gradient $C_{0}(t) \sim$ $A(t)^{\alpha / \ln 2}$ with $\alpha \simeq 50 \%$. What this means is that the size of the tissue doubles (i.e., cells divide) whenever Dpp signaling increases by $50 \%$. This quantitative relationship was conserved and found also in other imaginal discs, as well as in mutants of growth and signaling (Wartlick et al. 2011b). This also holds when the rate at which the increase of $50 \%$ is attained is exogenously manipulated by Dpp-signaling driven by applying a drug (Wartlick et al. 2011b), suggesting a causal relationship rather than a mere correlation. Based on these observations, a mechanism of growth control was proposed by which cells undergo mitosis when they detect an increase by $50 \%$ in Dpp signaling from the beginning of the cell cycle. Cells divide as long as they see such increase and cells stop dividing (the tissue does not proliferate anymore) when they no longer detect such an increase. It is still unclear how cells compute, beyond the absolute levels of signaling, the relative temporal increases in signaling levels to realize the growth rule with the characteristic relative increase $\alpha$. In other signaling systems, including chemotaxis in bacteria, sperm, and Caenorhabditis elegans (Barkai and Leibler 1997; Alon et al. 1999; Bargmann 2006; Friedrich and Jülicher 2007), it was shown that adaptive signaling can naturally lead to a detection of relative rates of change. Thus, growth control by temporal changes of Dpp could be naturally implemented by a Dpp signaling system that adapts to incoming stimuli (Wartlick et al. 2011a).

Therefore, control of degradation at the endocytic pathway mediates gradient scaling, which in turn leads to the increase of the amplitude of the gradient. Because of this expansion of the gradient, cells see an increase in Dpp signaling as the tissue grows. The perception of the temporal increase of signaling is, in turn, used by cells to decide when to divide and when to stop dividing. Specifically, these arguments suggest that the relative rate of increase $\dot{C} / C$ of the Dpp levels stimulates growth proportionally. Therefore, ultimately, it is the fine tissue-sizedependent control of endosomal dynamics that mediates the regulation of tissue proliferation and control of the final size (and shape?) of a developing organ.

\section{CONCLUDING REMARKS AND FUTURE PERSPECTIVES}

In the future, the quantitative analysis of endosomal network dynamics could be used to investigate the dynamics of cellular signaling pathways and the role of endosomal network dynamics for signaling. Receptors in the cell membrane that bind specific ligands are often internalized by endocytosis in small vesicles that subsequently fuse with endosomes. Thus, the signaling process depends on endosomal trafficking. Because of the role of endosomes as carriers of receptors that trigger a signaling cascade in the cell, signaling activity could influence endosomal kinetic parameters. Similarly, endosomal dynamics might influence the kinetics of signaling. Such coupling could be part of the dynamics of signaling pathways and would provide mechanisms for signaling pathways to regulate diverse cellular processes.

The theoretical analysis of the endocytic pathway and the formation and interpretation of morphogen gradients during patterning and growth has allowed the field to link different levels of complexity: trafficking of morphogens within the cell, their transport between cells, the formation of a gradient at the tissue level, and growth at the organ level. Theory shed light on what needs to be measured and this, in turn, has given the field an impulse to measure a quantitative dimension. Now we need to refine our theories, but also to refine our quantitative methods to measure the key parameters (e.g., growth, diffusion, turnover, binding/unbinding kinetics) more precisely. The system is not cracked, but (perhaps) the field has now the right approaches. 


\section{REFERENCES}

${ }^{*}$ Reference is also in this collection.

Affolter M, Basler K. 2007. The Decapentaplegic morphogen gradient: From pattern formation to growth regulation. Nat Rev Genet 8: 663-674.

Alon U, Surette MG, Barkai N, Leibler S. 1999. Robustness in bacterial chemotaxis. Nature 397: 168-171.

Bargmann CI. 2006. Chemosensation in C elegans WormBook: The online review of C elegans biology, WormBook, Pasadena, CA, pp. 1-29.

Barkai N, Leibler S. 1997. Robustness in simple biochemical networks. Nature 387: 913-917.

Belenkaya TY, Han C, Yan D, Opoka RJ, Khodoun M, Liu H, Lin X. 2004. Drosophila Dpp morphogen movement is independent of dynamin-mediated endocytosis but regulated by the glypican members of heparan sulfate proteoglycans. Cell 119: 231-244.

Ben-Zvi D, Barkai N. 2010. Scaling of morphogen gradients by an expansion-repression integral feedback control. Proc Natl Acad Sci 107: 6924-6929.

Ben-Zvi D, Pyrowolakis G, Barkai N, Shilo BZ. 2011. Expansion-repression mechanism for scaling the Dpp activation gradient in Drosophila wing imaginal discs. Curr Biol 21: 1391-1396.

Ben-Zvi D, Shilo BZ, Fainsod A, Barkai N. 2008. Scaling of the BMP activation gradient in Xenopus embryos. Nature 453: 1205-1211.

* Bökel C, Brand M. 2014. Endocytosis and signaling during development. Cold Spring Harb Perspect Biol doi: 10.1101/cshperspect.a017020.

Bollenbach T, Kruse K, Pantazis P, Gonzalez-Gaitan M, Jülicher F. 2005. Robust formation of morphogen gradients. Phys Rev Lett 94: 018103.

Bollenbach T, Kruse K, Pantazis P, Gonzalez-Gaitan M, Jülicher F. 2007. Morphogen transport in epithelia. Phys Rev E 75: 011901.

Christoforidis S, McBride HM, Burgoyne RD, Zerial M. 1999. The Rab5 effector EEA1 is a core component of endosome docking. Nature 397: 621-625.

* Di Fiore PP, von Zastrow M. 2014. Endocytosis, signaling and beyond. Cold Spring Harb Perspect Biol doi: 10.1101/ cshperspect.a016865.

Dunn KW, McGraw TE, Maxfield FR. 1989. Iterative fractionation of recycling receptors from lysosomally destined ligands in an early sorting endosome. J Cell Biol 109: 3303-3314.

Entchev E, Schwabedissen A, Gonzalez-Gaitan M. 2000. Gradient formation of the TGF-beta homolog Dpp. Cell 103: 981-991.

Foret L, Dawson JE, Villasenor R, Collinet C, Deutsch A, Brusch L, Zerial M, Kalaidzidis Y, Jülicher F. 2012. A general theoretical framework to infer endosomal network dynamics from quantitative image analysis. Curr Biol 22: 1381-1390.

Friedrich BM, Jülicher F. 2007. Chemotaxis of sperm cells. Proc Natl Acad Sci 104: 13256-13261.

* Gautreau A, Oguievetskaia K, Ungermann C. 2014. Function and regulation of the endosomal fusion and fission
Endocytosis during Morphogenetic Signaling

machineries. Cold Spring Harb Perspect Biol doi: 10.1101/ cshperspect.a016832.

Gonzalez-Gaitan M. 2003. Signal dispersal and transduction through the endocytic pathway. Nat Rev Mol Cell Biol 4: 213-224.

Hamaratoglu F, de Lachapelle AM, Pyrowolakis G, Bergmann S, Affolter M. 2011. Dpp signaling activity requires Pentagone to scale with tissue size in the growing Drosophila wing imaginal disc. PLoS Biol 9: e1001182.

Horiuchi H, Lippe R, McBride HM, Rubino M, Woodman P, Stenmark H, Rybin V, Wilm M, Ashman K, Mann M, et al. 1997. A novel Rab5 GDP/GTP exchange factor complexed to Rabaptin-5 links nucleotide exchange to effector recruitment and function. Cell 90: 1149-1159.

* Johannes L, Wunder C, Bassereau P. 2014. Bending "on the rocks"-A cocktail of biophysical modules to build endocytic pathways. Cold Spring Harb Perspect Biol 6: a016741.

Kicheva A, Pantazis P, Bollenbach T, Kalaidzidis Y, Bittig T, Jülicher F, Gonzalez-Gaitan M. 2007. Kinetics of morphogen gradient formation. Science 315: 521-525.

Kicheva A, Bollenbach T, Wartlick O, Jülicher F, GonzalezGaitan M. 2012. Investigating the principles of morphogen gradient formation: From tissues to cells. Curr Opin Genet Dev 22: 527-532.

* Klumperman J, Raposo G. 2014. The complex ultrastructure of the endolysosomal system. Cold Spring Harb Perspect Biol doi: 10.1101/cshperspect.a016857.

Kruse K, Pantazis P, Bollenbach T, Jülicher F, Gonzalez-Gai$\tan$ M. 2004. Dpp gradient formation by dynamin-dependent endocytosis: Receptor trafficking and the diffusion model. Development 131: 4843-4856.

* Mayor S, Parton RG, Donaldson JG. 2014. Clathrin-independent pathways of endocytosis. Cold Spring Harb Perspect Biol doi: 10.1101/cshperspect.a016758.

Mellman I. 1996. Endocytosis and molecular sorting. Ann Rev Cell Dev Biol 12: 575-625.

* Merrifield CJ, Kaksonen M. 2014. Endocytic accessory factors and regulation of clathrin-mediated endocytosis. Cold Spring Harb Perspect Biol doi: 10.1101/cshper spect.a016733.

Rink J, Ghigo E, Kalaidzidis Y, Zerial M. 2005. Rab conversion as a mechanism of progression from early to late endosomes. Cell 122: 735-749.

Rusakov DA, Kullmann DM. 1998. Geometric and viscous components of the tortuosity of the extracellular space in the brain. Proc Natl Acad Sci 95: 8975-8980.

Simonsen A, Lippe R, Christoforidis S, Gaullier JM, Brech A, Callaghan J, Toh BH, Murphy C, Zerial M, Stenmark H. 1998. EEA1 links $\mathrm{PI}_{3} \mathrm{~K}$ function to Rab5 regulation of endosome fusion. Nature 394: 494-498.

Spemann H, Mangold H. 1924. Über induktion von Embryonalagen durch Implantation Artfremder Organisatoren. Roux' Arch Entw Mech 100: 599-638.

Taylor MJ, Perrais D, Merrifield CJ. 2011. A high precision survey of the molecular dynamics of mammalian clathrin-mediated endocytosis. PLoS Biol 9: e1000604.

Teleman AA, Cohen SM. 2000. Dpp gradient formation in the Drosophila wing imaginal disc. Cell 103: 971-980.

Turing A. 1952. The chemical basis of morphogenesis. Phil Trans R Soc Lond 237: 37-72. 
M. Gonzalez-Gaitan and F. Jülicher

van der Goot FG, Gruenberg J. 2006. Intra-endosomal membrane traffic. Trend Cell Biol 16: 514-521.

Vuilleumier R, Springhorn A, Patterson L, Koidl S, Hammerschmidt M, Affolter M, Pyrowolakis G. 2010. Control of Dpp morphogen signalling by a secreted feedback regulator. Nat Cell Biol 12: 611-617.

* Wandinger-Ness A, Zerial M. 2014. Rab proteins and the compartmentalization of the endosomal system. Cold Spring Harb Perspect Biol doi: 10.1101/cshperspect.a022616.

Wartlick O, Mumcu P, Jülicher F, Gonzalez-Gaitan M. 2011a. Understanding morphogenetic growth controlLessons from flies. Nat Rev Mol Cell Biol 12: 594-604.

Wartlick O, Mumcu P, Kicheva A, Bittig T, Seum C, Julicher F, Gonzalez-Gaitan M. 2011b. Dynamics of Dpp signaling and proliferation control. Science 331: 1154-1159.
Wolpert L. 1969. Positional information and the spatial pattern of cellular differentiation. J Theor Biol 25: 147.

Yu SR, Burkhardt M, Nowak M, Ries J, Petrasek Z, Scholpp S, Schwille P, Brand M. 2009. Fgf8 morphogen gradient forms by a source-sink mechanism with freely diffusing molecules. Nature 461: 533-536.

Zecca M, Basler K, Struhl G. 1996. Direct and long-range action of a wingless morphogen gradient. Cell 87: 833844.

Zerial M, McBride H. 2001. Rab proteins as membrane organizers. Nat Rev Mol Cell Biol 2: 107-117.

Zhou S, Lo WC, Suhalim JL, Digman MA, Gratton E, Nie Q, Lander AD. 2012. Free extracellular diffusion creates the Dpp morphogen gradient of the Drosophila wing disc. Curr Biol 22: 668-675. 


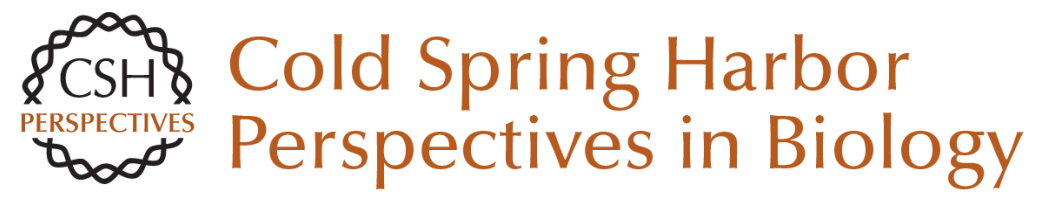

\section{The Role of Endocytosis during Morphogenetic Signaling}

Marcos Gonzalez-Gaitan and Frank Jülicher

Cold Spring Harb Perspect Biol 2014; doi: 10.1101/cshperspect.a016881

Subject Collection Endocytosis

\section{Endocytosis: Past, Present, and Future} Sandra L. Schmid, Alexander Sorkin and Marino Zerial

Rab Proteins and the Compartmentalization of the Endosomal System Angela Wandinger-Ness and Marino Zerial

Cargo Sorting in the Endocytic Pathway: A Key Regulator of Cell Polarity and Tissue Dynamics Suzanne Eaton and Fernando Martin-Belmonte

Unconventional Functions for Clathrin, ESCRTs, and Other Endocytic Regulators in the Cytoskeleton, Cell Cycle, Nucleus, and Beyond: Links to Human Disease

Frances M. Brodsky, R. Thomas Sosa, Joel A. Ybe, et al.

Endocytosis of Viruses and Bacteria Pascale Cossart and Ari Helenius

Lysosomal Adaptation: How the Lysosome Responds to External Cues Carmine Settembre and Andrea Ballabio

Reciprocal Regulation of Endocytosis and Metabolism

Costin N. Antonescu, Timothy E. McGraw and Amira Klip

Endocytosis and Autophagy: Exploitation or Cooperation?

Sharon A. Tooze, Adi Abada and Zvulun Elazar
Imaging and Modeling the Dynamics of

Clathrin-Mediated Endocytosis Marcel Mettlen and Gaudenz Danuser

Endocytic Accessory Factors and Regulation of Clathrin-Mediated Endocytosis

Christien J. Merrifield and Marko Kaksonen

The Complex Ultrastructure of the Endolysosomal System Judith Klumperman and Graça Raposo

The Biogenesis of Lysosomes and Lysosome-Related Organelles J. Paul Luzio, Yvonne Hackmann, Nele M.G. Dieckmann, et al.

Endocytosis, Signaling, and Beyond Pier Paolo Di Fiore and Mark von Zastrow

Clathrin-Independent Pathways of Endocytosis Satyajit Mayor, Robert G. Parton and Julie G. Donaldson

The Role of Endocytosis during Morphogenetic Signaling Marcos Gonzalez-Gaitan and Frank Jülicher

Role of Endosomes and Lysosomes in Human Disease

Frederick R. Maxfield

For additional articles in this collection, see http://cshperspectives.cshlp.org/cgi/collection/

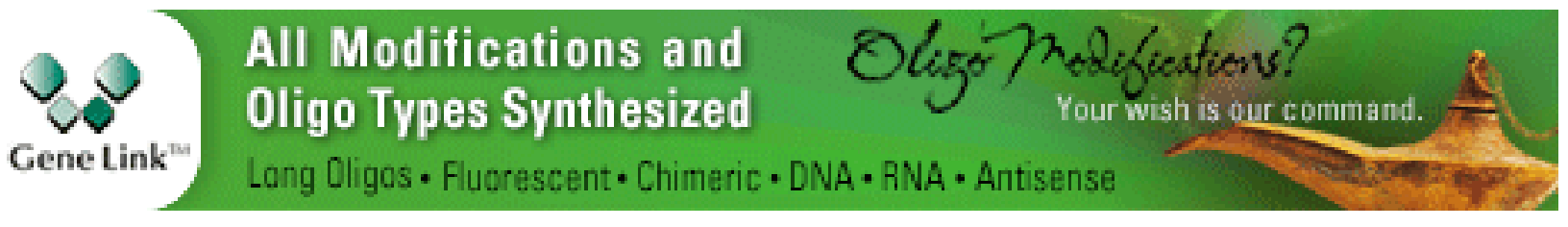


For additional articles in this collection, see http://cshperspectives.cshlp.org/cgi/collection/

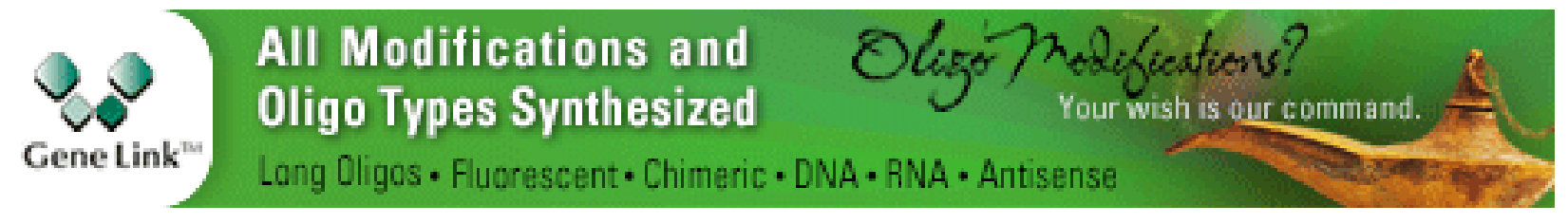

Copyright @ 2014 Cold Spring Harbor Laboratory Press; all rights reserved 